Journal thinks the New York commissioner of stree cleaning, Dr. Woodbury, did well to brine the matter "Iorcibly forward in a recent address to the "Civic Club. throw shoes, oilcloths, and everything else right out on consequence a growing ground is afforded for the worst
possible diseases. Four hundred and twelve sweepers are now on the sick report with bronchial diseases and Refuse not only serves as a nidu matic cleansing that the gutters are designed to accom plish, and it increases the difficulty of the sweepers

[Continued from SUPPLEMENT No. 1396, page 22371.] HISTORY OF COLD AND THE ABSOLUTE ZERO.* By Prof. James Dewar

IT will be apparent from what has just been said
hat, thanks to the labors of Andrews, van der Waals, and others, theory had again far outrun experiment. We could calculate the constants and predict some of the simple physical characteristics of liquid oxygen,
hydrogen or nitrogen with a high degree of confidence long before any one of the three had been obtained in
the static liquid condition permitting of the experimental verification of the theory. This was the more
tantalizing, because, with whatever confidence the tantalizing, because, with whatever confidence the
chemist may anticipate the substantial corroboration of his theory, he also anticipates with almost equal conviction that, as he approaches more and more nearly to the zero of absolute temperature, he will
encounter phenomena compelling modification, revision and refinement of formulas which fairly covered
the facts previously known. Just as nearly seventy years ago chemists were waiting for some means of
getting a temperature of 100 degrees below melting ice, so ten years ago they were casting about for the it need haraly be said, increases in a geometrical may be estimated from the fact that to produce liquid air in the atmosphere of an ordinary laboratory is a feat analogous to the production of liquid water
starting from steam at a white heat and working with all the implements and surroundings at the same high produce intense cold as how to save it when produced from being immediately leveled up by the relatively packings were inadmissible because they are both
cumbrous and opaque, while in working near the limits of our resources it is essential that the product should
be visible and readily handled. It was while puzzling over this mechanical and manipulative difficulty in
1892 that it occurred to me that the principle of an arrangement used nearly twenty years before in some calorimetric experiments, which was based upon the employed with advantage as well to protect old substances from heat as hot ones from rapid cooling. I tween being very highly exhausted. Experiments showed that liquid air evaporated at only one-fifth of unexhausted vessel, owing to the convective transferreduced by the high vacuum. But, in addition, these
vessels lend themselves to an arrangement by which vessels lend themselves to an arrangement by which
radiant heat can also be cut off. It was found that posit of silver the influx of heat was diminished to one-sixth the amount entering without the metallic coating. The total effect of the high vacuum and the cent. The efficiency of such vessels depends upon of the best means of effecting the desired exhaustion. has to be exhausted with an easily condensable vapor the primary vessel that can be sealed off. The ad vantage of this method is that no air pump is required of exhaustion that can be obtained. The action is
rapid, provided liquid air is the cooling agent, and rapid, provided liquid air is the cooling agent, and
vapors like mercury, water or benzol are employed.
It is obviouls that when we have to deal with such an exceptionally volatile liquid as hydrogen, the vapor filling may be omitted because air itself is now an easily condensable vapor. In other words, liquid
hydrogen, collected in such vessels with the annular
space full of air, immediately solidifies the air and space full of air, immediately solidifies the air and
thereby surrounds itself with a high vacuum. In the boiling on the absolute scale at about 5 degrees, as might have the annular space filled with the latter gas to begin with, and yet get directly a very high
vacuum, owing to the solidification of the hydrogen. Nany combinations of vacuum vessels can be arranged, operate the more useful they become. Vessels of this kind are now in general use, and in them liquid air
has crossed the American continent. Of the various forms, that variety is of special importance which so that any liquid gas may be drawn off from the interior of such a vessel. In the working of regenera-
tive coils such a device becomes all-important, and
such special vessels cannot be dispensed with for the
liquefaction of hydrogen. In the early experiments of Pictet and Cailletet
cooling was produced by the sudden expansion of the highly compressed gas, preferably at a low temperature, the former using a jet that lasted for some time, strong glass tube. Neither process was practicable as a mode of producing liquid gases, but both gave valu-

*Abstract of a Presidential Address delivered at the Belfast meeting on
September 10, 1902, of the British A\&sociation for the Advancement of by the production of a temporary mist. Linde, how-
ever, saw that the continuous use of a jet of highly compressed gas, combined with regenerative cooling the Kelvin-Joule effect; and he succeeded in making a machine, based on this principle, capable of producing ers had proved that, owing to molecular attraction corspressed gases passing through a porous plug or amount depending on the difference of pressure, and nversely as the square of the absolute temperature The meoling is greater a steady difference of pressure only gas that did not show cooling under such con-
ditions was hydrogen. Instead of being cooled it ditions was hydrogen. Instead of being cooled it
became actually hotter. The reason for this apparent anomaly in the Kelvin-Joule effect is that every gas is heated and below which it is cooled. This inver three-quarter times the critical point. The efficiency compressed gas well below the inversion temperature, place of the critical one, when in the ordinary way specific liquid cooling agents. The success of both temperature range, only the Linde method gives us a much wider range of temperature within which lique-
faction can be effected. This is not the case if, instead of depending on getting cooling by the internal work the compressed gas to do external work as in the
well-known air machines of Kirk and Coleman. Both these inventors have pointed out that there is no
limit of temperature, short of liquefaction of the gas in use in the circuit, that such machines are not ca-
pable of giving. While it is theoretically clear that such machines ought to be capable of maintaining penditure of power, it is a very, different matter to overcome the practical difficulties of working such machines under the conditions. Coleman kept a machine delivering air at minus 83 degrees for hours, but he Monsieur Claude, of Paris, has, however, succeeded in
working a machine of this type so efficiently that he working a machine of this type so efficiently that he
has managed to produce one liter of liquid air per horse power expended per hour in the running of the
engine. This output is twice as good as that given engine. This output is twice as good as that given
by the Linde machine, and there is no reason to doubt that the yield will be still further improved. It is production of liquid air and hydrogen will be effected cold by the expenditure of mechanical work.

\section{LIQUID HYDROGEN AND HELIUM.}

To the physicist the copious production of liquid air
by the methods described was of peculiar interest and value as affording the means of attacking the far more difficult problem of the liquefaction of hydrogen, and even as encouraging the hope that liquid hydrogen
might in time be employed for the liquefaction of yet more volatile elements, apart from the importance which its liquefaction must hold in the process of
the steady advance toward the absolute zero. Hydrogen is an element of especial interest, because the
study of its properties and chemical relations led and Andrews to entertain the view that if it could ever be brought into the state of liquid or solid it would reveal metallic characters. Looking to the
special chemical relations of the combine hydrogen in water, alkaline oxides, acids and salts, together
with the behavior of these substances on electrolysis, we are forced to conclude that hydrogen behaves as ery of Graham that palladium can absorb some hun dreds of times its own volume of hydrogen and still
retain its luster and general metallic character, the impression that hydrogen was probably a member of
the metallic group became very general. The only chiemist who adopted another view was my distin-
guished predecessor, Prof. Odling. In his "Manual of Chemistry," published in 1861, he pointed out that
hydrogen has chlorous as well as basic relations, and that they are as decided, important, and frequent as
its other relations. From such considerations he arrived at the conclusion that hydrogen is essentially a neutral or intermediate body, and therefore we
should not expect to find liquid or solid hydrogen
possess the appearance of a metal. This extraordinary possess the appearance of a metal. This extraordinary Another curious anticipation was made by Dumas in a letter addressed to Pictet, in which he says that and that probably both elements have the same, atomic volume, so that the density of hydrogen, for this
reason, would be about the value elicited by subsereason, would be about the value elicited by subse-
quent experiments. Later on, in 1872, when Newlands began to arrange the elements in periodic groups, he rine family; but Mendeléeff in his later classification placed hydrogen in the group of the alkaline metals;
on the other hand, Dr. Johnstone Stoney classes hydrogen with the alkaline earth metals and magnesium. conclusion could be reached regarding the physical
properties of liquid or solid hydrogen, and the only
way to arrive at the truth was to prosecute low-tem perature research until success attended the efforts to produce its liquefaction. This result I definitely obtained in 1898. The case of liquid hydrogen is, in
fact, an excellent illustration of the truth already
referred to, that no theoretical forecast, however apreferred to, that no theoretical forecast, however ap-
parently justified by analogy, can be finally accepted parently justified by analogy, can be finally accepted
as true until confirmed by actual experiment. Liquid
hydrogen is a colorless, transparent body of extraor hydrogen is a colorless, transparent body of extraor-
dinary intrinsic interest. It has a clearly defined fact that its surface tension is only the thirty-ifth part of that of water, or about one-fifth that of liquid
air, and can be pourea easily from vessel to vessel.
The liquid does not conduct electricity, and, if any-
thing, is slightly diamagnetic. Compared with an
equal volume of liquid air, it requires only one-fifth the quantity of heat for vaporization; on the other
hand, its specific heat is ten times that of liquid air or five times that of water. The coefficient of expansion of the fluid is remarkable, being about ten times
that of gas; it is by far the lightest liquid known to exist, its density being only one-fourteenth that of
water; the lightest liquid jreviously known was liquid marsh gas, which is six times heavier. The only solid which has so small density as to float upon its sur-
face is a piece of pith wood. It is by far the coldest liquid known. At ordinary atmospheric pressure it The. critical point of the liquid is about 29 degrees
absolute and the critical pressure not more than fifteen the liquid has nearly the density of air - that is, it is ature. Reduction of the pressure by an air pump
brings down the temperature to minus 258 degrees, when the liquid becomes a solid resembling frozen foam, and this by further exhaustion is cooled to
minus 260 degrees, or 13 degrees absolute, which is the lowest steady temperature that has been reached. parent ice, melting at about 15 degrees absolute, under a pressure of 55 millimeters, possessing the unique density of one-eleventh that of water. Such cold in-
volves the solidification of every gaseous substance but one that is at present definitely known to the chemist, and so liquid hydrogen introduces the investigator to refrigerating substance and liquid air is most remark-
able. On the removal of the loose plug of cotton-wool used to cover the mouth of the vacuum vessel in which
it is stored, the action is followed by a miniature snowstorm of solid air, formed by the freezing of the with the cold vapor rising from the liquid. This
solid air falls into the vessel and accumulates as a When the outside of an ordinary test tube is cooled by immersion in the liquid, it is soon observed to fill a with solid air, and if the tube be now lifted out both in the inside and on the outside of the tube-in other by condensation from the atmosphere. A tuft
of cotton-wool soaked in the liquid and then held near be inferred therefrom that liquid hydrogen is a mag. netic body. This, however, is not the case: the attrac-
tion is due neither to the cotton-wool nor to the hydrogen-which indeed evaporates almost as soon as the the air, which is well known to be a magnetic body, The strong condensing powers of liquid hydrogen afford a simple means of producing vacua of very high ordinary air is placed for a short time in the liquitit,
the contained air accumulates as a solid at the bottom. whlle the higher part is almost entirely deprived of
particles of gas. So perfect is the vacuum thus formed that the electric discharge can be made to pass only with the greatest difficulty. Another important ap-
plication of liquid air, lituid hydrogen, etc., is as analytic agents. Thus, if a gaseous mixture be cooled by means of liquid oxygen, only those constituents densable than oxygen. Similarly, if this gaseous residue be in its turn cooled in liquid hydrogen, a still
further separation will be effected, everything that is less volatile than hydrogen being condensed to a liquid
or solid. By proceeding in this fashion it has been or solid. By proceeding in this fashion it has been one thousand. By the evaporation of solid hydrogen oner the air pump we can reach within 13 or 14 degrees of the zero, but there or thereabouts our
progress is barred. This gap of 13 degrees might seem at first sight incignificant in comparison with to win one degree low down the scale is quite a different matter from doing so at higher temperatures; would be a far greater achievement than any so far would be a far greater achievement than any so far
accomplished in low-temperature research. For the
difficulty is twofold, having to do partly with process and partly with material. The application of the methods used in the liquefaction of gases becomes con-
tinually harder and more troublesome as the working to liquid hydrogen-a difference of 60 degrees-is, from a thermodynamic point of view, as difficult as to bridge the gap of 150 degrees that separates liquic
chloride and liquid air. By the use of a new liquid
gas exceeding hydrogen in volatility to the same exgas exceeding hydrogen in volatility to the same ex-
tent as hydrogen does nitrogen, the investigator might get to within five degrees of the zero; but even
second hypothetical substance, again exceeding th first one in volatility to an equal extent, would not That the zero will ever be reached by man is extremely improbable. A thermometer introduced into regions phere might approach the absolute zero, provided that
its parts were highly transparent to all kinds of radia. tion, otherwise it would be affected by the radiation of
the sun, and would therefore become heated. But supposing all difficulties to be overcome, and the experizero, it is by no means certain that he would find the near approach of the death of matter sometimes pic-
tured. Any forecast of the phenomena that would be seen must be based on the assumption that there is temperatures and those which take place at still lower
ones. Is such an assumption justified? It is true tha ${ }^{+}$ ones. Is such an assumption justified? It is true tha ${ }^{f}$ which they are exposed. But it would be rash to take for granted that the changes which have been traced in explored regions continue to the same extent and in
the same direction in those which are as yet unes- 
plored. Of sucic a breakdown low-temperature re
search has already yielded a direct proof at least in one case. A series of experiments with pure metals showed they are electrical resistance gradually decreases as such ratio that it appeared probable that at the zero of absolute temperature they would have no resistance at all and would become perfect conductors of elec-
tricity. This was the inference that seemed justifiable by observations taken at depths of cold which can be obtained by means of liquid air and less powerful re frigerants. But with the advent of the more powerfu revise that conclusion. A discrepancy was first observed when a platinum resistance thermometer was
used to ascertain the temperature of that liquid boil ing under atmospheric and reduced pressure. All being placed in the exhausted receiver of an air pump. undergo a reduction in temperature, but when hydro gen was treated in this way it appeared to be an
exception. The resistance thermometer showed no re auction as was expected, and it became a question was behaving ainormally. Ultimately, by the adoption of the hydrogen was proved to be lowered by exhaustion as theory indicated. Hence it was the platinum thermometer which had broken down; in other word
the electrical resistance of the metal employed in its construction was not, at temperatures about minus
250 deg. C., decreased by cold in the same proportion
as at temperatures about minus 200 deg. This being as at temperatures about minus $200 \mathrm{deg}$. This being
the case, there is no longer any reason to suppose that conductor of electricity; and in view of the similarity between the behavior of platinum and that of othe tivity, the presumption is that the same is true of them
also. At any rate the knowledge that in the case of at also. At any rate the knowledge that in the case of at
least one property of matter we have succeeded in attaining a depth of cold sufficient to bring about un expected change in the law expressing the variation
of that property with temperature, is sufficient to show the necessity for extreme caution in extending our inferences regarding the properties of matter nea the zero of temperature. Lord Kelvin evidently an-
ticipates the possibility of more remarkable electrical ticipates the possibility of more remarkable electrical
properties being met with in the metals near the zero. properties being met with in the metals near the "ero. cal metal having the following remarkable properties: below 1 degree absolute it is a perfect insulator of electricity, at 6 degrees it possesses high conductivity.
tivity, and at 6 de safely be predicted that liquid hydrogen will be the means by which many obscure problems of physics and chemistry will ultimately be solved, so
that the liquefaction of the last of the old permanent gases is as pregnant now with future consequences of great scientific moment as was the liquefaction
chlorine in the early years of the last century.

The next step toward the absolute zero is to find another gas more volatile than hydrogen, and that we Ramsay as helium, a gas which is widely distributed,
like hydrogen, in the sun, stars and nebula. A specilike hydrogen, in the sun, stars and nebulæ. A speci-
men of this gas was subjected by Olszewski to liquid air temperatures, combired with compression and subresulted in his being unable to discover method, an ance of liquefaction, even in the form of mist. His experiments led him to infer that the boiling point of the substance is probably below 9 degrees absolute.
After Lord Rayleigh had found a new source of helium
in the gases which are derived from the Bath springs, in the gases which are derived from the Bath springs, and liquid hydrogen became available as a cooling showed the formation of fluid, but this turned out to
be owing to the presence of an unknown admixture of other gases. As a matter of fact, a year before the date of this experiment I had recorded indications of
the presence of unknown gases in the spectrum of
helium derive from this source. When subsequently such condensable constituei is were removed, the puri-
fied helium showed no signs of liquefaction, even when fied helium showed no signs of liquefaction, even when
compressed to 80 atmospheres, while the tube con
taining it was surrounded with solid hydrogen. Further, on suddenly expanding, no instantaneous mist appeared. Thus helium was definitely proved to be a much more volatile substance than hydrogen in either drawn from the adiabatic expansion effected under the circumstances is that helium must have touched a tem-
perature of from 9 to 10 degrees for a short time without. showing any signs of liquefaction, and conseThis would force us to anticipate that the boiling point helium will be four times more volatile than liquid hydrogen. just as liquid hydrogen is four times more the gas is a problem for the future, this does not pre-
vent us from safely anticipating some of the properties vent us from safely anticipating some of the properties
of the fluid body. It would be twice as dense as liquid mospheres. The liquid would possess a very feeble surface-tension, and its compressibility and expansibil while the heat required to vaporize the molecule would
be about one-fourth that of liquid hydrogen. Heatins the liquid 1 degree above its boiling point would raise the pressure by $1 \% / 4$ atmospheres, which is more than
four times the increment for liquid hydrogen. The liquid would be only seventeen times denser than its vapor, whereas liquid hydrogen is sixty-five times denser than the gas it gives off. Only some 3 or 4
degrees would separate the critical temperature from the boiling point and the melting point, whereas in
liquid hydrogen the separation is respectively 10 and nitrogen and hydrogen are closely proportional to the gaseous values, and as Lord Rayleigh has shown that gen, although it is twice as dense, we must infer thai
the refractivity of liquid helium would also be about the refractivity of liquid helium would also be about
one-fourth that of liquid hydrogen. Now hydrogen has the smallest refractivity of any known liquid, and
yet liquid helium will have only about one-fourth o this value-comparable, in fact, with liquid hydrogen liquid will be quite exceptional in its optical proper
ties, and very difficult to see. This may be the ex planation of why no mist has been seen on its adiabatic expansion from the lowest temperatures. Taking all these remarkable properties of the liquid into con present able to cope with the difficulties involved in its production and collection. Provided the critical from the knowledge of the conditions that are succes ful in producing a change of state in hydrogen through
the use of liquid air, we may safely predict that helithe use of liquid air, we may safely predict that heli
um can be liquefied by following similar methods. If however, the critical point is as low as 6 degrees abso-
lute, then it would be almost hopeless to anticipate success by adopting the process that works so well with hydrogen. The present anticipation is that the only, instead of liquid air under exhaustion being used
as the primary cooling agent, liquid hydrogen evapoas the primary cooling agent, liquid hydrogen evapo-
rating under similar circumstances must be employed. In this case, the resulting liquid would require to be
collected in a vacuum vessel the outer walls of which are immersed in liquid hydrogen. The practical difficulties and the cost of the operation will be very
great; but, on the other hand, the descent to a tem perature within 5 degrees of the zero would open out new vistas of scientific inquiry, which would add im
mensely to our knowledge of the properties of matter. mensely to our knowledge of the properties of matter
To command in our laboratories a temperature which would be equivalent to that which a comet might deed be a great triumph for science. If the present Royal Institution attack on helium fail, then we must ultimately succeed by adopting a process based on th ance of external work. When a turbine can be worked by compressed helium, the whole of the mechanism
and circuits being kept surrounded with liquid hydrowill be effected. "In all probability gases other than helium will be discovered of greater volatility than hydrogen. It was at the British Association Meeting
in 1896 that I made the first suggestion of the probable existence of an unknown element which would he
found to fill up the gap between argon and helium, and this anticipation was soon taken up by others and
ultimately confirmed. Later, in the Bakerian Lecture for 1901, I was led to infer that another member of weight about 2 , and this would give us a gas still more volatile, with which the absolute zero might be still such element or elements may yet be isolated and
identified as coronium or nebulium. If among the unknown gases possessing a very low critical point one, which ordinary experience would lead us to an
ticipate, then such difficultly liquefiable gases would produce fluids having different physical properties from any of those with which we are acquainted. and densities than hydrogen, yet all such gases must according to our present views of the gaseous state, be capable of liquefaction before the zero of tempera-
ture is reached. The chemists of the future will find ample scope for investigation within the apparently
imited range of temperature which separates solid hydrogen from zero. Indeed, great as is the sentimental interest attached to the liquefaction of these refractory gases, the importance of the achievement
lies rather in the fact that it opens out new fields of research and enormously widens the horizon of physi-
cal science, enabling the natural philosopher to study the properties and behavior of matter under entirely yet only in its infancy, but speedy and extensive developments may be loked for, since within recent a liquid-air plant is becoming a common adjunct to a liquid-air plant is becoming a common
the equipment of the ordinary laboratory.

\section{THE UPPER AIR AND AURORAS.}

The present liquid ocean, neglecting everything for of the earth's history part of the atmosphere, and its cooling of the earth's surface. This resulting ocean is subjected to the pressure of the remaining uncon-
densed gases, and as these are slightly soluble they dissolve to some extent in the fluid. The gases in hausting the water, and if we compare their volume with the volume of water as steam, we should find about 1 volume of air in 60,000 volumes of steam.
This. would then be about the rough proportion of the
relatively permanent gas to condensable gas which relatively permanent gas vaporized ocean. Now let us assume the surface of the earth gradually cooled
to some 200 degrees below the freezing point; then, after all the present ocean was frozen, and the climate frost, a new ocean of liquid air would appear, covering
the entire surface of the frozen globe about thirty-five feet deep. We may now apply the same reasoning water one, and this would lead us to anticipate that
it might contain in solution some gases that may be fluid. In order to separate them we must imitate the method of taking the gases out of water. Assume a mample of liquid air cooled to the low temperature that pipe to a condenser cooled in liquid hydrogen; then with the first portions of the air, and can be pumped off, being uncondensable at the temperature of the the known gases, free hydrogen, helium and neon, has been separated from liquid air. It is interesting to
note in passing that the relative volatilities of water
and oxygen are in the same ratio as those of liquid air and hydrogen, so that the analogy between the ocean
of water and that of liquid air has another suggestive parallel. The total uncondensable gas separated in this way amounts to about one fifty-thousandth of the volume of the air, which is about the same proportion
as the air dissolved in water. That free hydrogen exists in air in small amount is conclusively proved,
but the actual proportion found by the process is very much smaller than Gautier has estimated by the com leigh, show that Gautier, who estimated the hydrogen present as one five-thousandth, has in some way pro duced more hydrogen than he can manage to extract The spectroscopic examination of these gases throws nature of the upper air. On passing electric dis-
charges through the tubes containing the most volatile orange light, which is especially marked at the negative pole. The spectroscope shows that this light con-
sists, in the visible part of the spectrum, chiefly of a succession of strong rays in the red, orange and yel-
low, attributed to hydrogen, helium and neon. Be sires these, a vast number of rays, generally less
brilliant, are distributed through the whole length of the visible spectrum. The greater part of these rays
are of, as yet, unknown origin. The violet and ultraviolet part of the spectrum rivals in strength that of include some of the gases that pervade interplanetary space, search was made for the prominent nebular,
coronal and auroral lines. No definite lines agreeing with the nebular spectrum could be found, but many lines occurred closely coincident with the coronal and
auroral spectrum. But before discussing the spectroscopic problem it will be necessary to consider the and condition of the upper a

$$
\text { ( To be continued.) }
$$

ON THE ATOMIC WEIGHT OF RADIUM. By Mdme. Curie.

By concentrating by fractional crystallization the greater part of the radiferous barium at $\mathrm{my}$ disposal $\mathrm{I}$ have succeeded in obtaining about one decigramme of
perfectly pure radium chloride. This has enabled me to determine the atomic weight of radium.

It results from the following experiments that the
atomic weight of radium is 225 ( taking $\mathrm{Cl}=35.4$ and $\mathrm{Ag}=107.8$ ) with a probable uncertainty of not more element. The method employed consists of estimating, in the
state of chloride of silver, the chlorine contained in a state of chloride of silver, the chlorine contained in a
known weight of anhydrous radium chloride. As a control experiment I determined the atomic weight of tions, and with the same quantity of material. The nave also found that this method gives satisfactory results even with a very small quantity of material. Many determinations were made with radium
chloride; after each operation the radium was brought liquid, containing after the estimation radium nitrate drochloric acid. The silver chloride was separated ness several times with an excess of pure hydrochloric
acid. Experience shows that all the nitric acid may be eliminated in this way.

The weighings were performed on an aperiodic Curie balance in perfect adjustment, and weighing to balance permits of very rapid weighings, an essential condition for weighing the anhydrous chlorides of barium and radium, which slowly absorb water in
spite of the presence of desiccating agents in the balance. The substance to be weighed is placed in a long time, and I have verified that its weight does operation.

The hydrated chloride obtained by crystallization hydroles chloride. Experience shows that when the chloride was kept for some hours at 100 deg. its weight deg. and is kept there for some hours. Therefore the
anhydrous chloride so obtained constitutes a perfectly
definite body. In all the estimates the chloride was dried at $150 \mathrm{deg}$

spectrum of the has been good enough to examine the radium chloride submitted to analysi of purity of the body.

Two series of experiments were made. The first
was with a radium chloride which M. Demarcay considered sensibly pure, but in which the spectrum sidered sensibly pure, but in which the spectrum
showed still the three principal lines of barium with cessive operations were the following:

$$
\begin{aligned}
& \text { ive operations were the following: } \\
& 220.7 \quad \therefore 223.0 \quad 222.8
\end{aligned}
$$

I then undertook a new purification, and succeeded In obtaining a product much more pure. M. Demar
cay considers that this second product only contains
a minimum quantity of barium incapable of influencing appreciably the atomic weight.

nations made 225.3 perfectly pure radium:

The mean of these numbers is 225.0 224.0

number is within one unit of the truth. ways radio-active and luminous. I satisfied myselthat it had not carried down a ponderable quantity of
radium. I have found also that the weight of radium radium. I have found also that the weight of radium The separation of the radium chloride was effecter by fractionally crystallizing in a hydrochloric solu-
tion a radiferous barium chloride which already had tion a radiferous barium chloride which already had
been purfied carefully. When the concentration of
radium becomes of a certain strength the crystals, at first colorless in the mother-liquor, become yellow or
rose colored $a$ few hour after their deposition. This 